\title{
Publication activity in the field of Sjögren's syndrome: a ten-year Web of Science based analysis
}

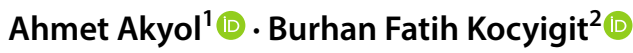

Received: 29 August 2020 / Accepted: 25 September 2020

(c) Springer-Verlag GmbH Germany, part of Springer Nature 2020

\begin{abstract}
Bibliometric analysis is widely utilized to evaluate global research productivity in different research topics. However, to date, there has been no assessment of worldwide research productivity associated with Sjögren's syndrome. Therefore, the aim of this study was to evaluate the global research productivity in the field of Sjögren's syndrome using bibliometric analysis. The Web of Science database was scanned with the search terms 'Sjögren's syndrome' and 'Sjögren syndrome' for publications in the period 2010-2019. Original articles and reviews were selected for analysis. The most active countries were determined, and the number of articles, citations, research productivity adjusted by population and gross domestic product were analyzed. A total of 3856 articles were identified from 65 different countries. A statistically significant trend was observed in the direction of increase (in terms of the number of articles; from 282 to 461 ) in the 10-year period. A total of $3004(77.90 \%)$ articles were from high-income countries. The five most productive countries were the United States $(n=714,18.51 \%)$, China $(n=428,11.09 \%)$, Japan $(n=308,7.98 \%)$, Italy $(n=299,7.75 \%)$ and France $(n=249,6.45 \%)$. When the number of articles was adjusted according to population, Norway was the most productive country, followed by Greece and the Netherlands. In analysis according to gross domestic product, Greece was the leading country, followed by Norway and the Netherlands. The results of this study demonstrated a remarkable growth in global research productivity on Sjögren's syndrome between 2010 and 2019. More than three quarters of the articles were from high-income countries. When population and gross domestic product were considered, relatively small European countries came to the fore.
\end{abstract}

Keywords Sjögren's syndrome $\cdot$ Bibliometric analysis $\cdot$ Articles $\cdot$ Publications $\cdot$ Web of Science

\section{Introduction}

Sjögren's syndrome (SS) is a systemic autoimmune disease that causes inflammation of the exocrine glands, primarily the salivary and lacrimal glands. This inflammatory process triggers dryness of mucosal surfaces and the development of sicca symptoms in the mouth and eyes [1]. SS, which predominantly affects females, is not limited to these effects and can cause systemic symptoms that mainly involve the

Burhan Fatih Kocyigit

bfk2701@ hotmail.com

Ahmet Akyol

ahmetakyol07@gmail.com

1 Physiotherapy and Rehabilitation Application and Research Center, Hasan Kalyoncu University, Gaziantep, Turkey

2 Faculty of Medicine, Department of Physical Medicine and Rehabilitation, Kahramanmaraş Sütçü İmam University, Kahramanmaraş, Turkey musculoskeletal system, lungs, liver, kidneys, nervous system, and vascular system [2, 3]. Epidemiological studies have reported confusing prevalence figures from $0.01 \%$ to more than 3\% for SS [4]. Difficulties in diagnosis of the disease may have led to these conflicting prevalence results [5].

Bibliometric research is a frequently used and wellestablished instrument for quantitatively and qualitatively assessing global academic productivity in specific areas [6]. Bibliometric analysis provides data on countries' academic productivity, increase/decrease and trend of articles and distribution of papers in terms of country and language [7]. It is an important tool allowing the development and patterns of various scientific subjects to be followed. It also provides useful data to increase resources transferred to slow-progressing or underdeveloped scientific fields and to create new medical policies [8].

Bibliometric studies have been performed on various diseases and topics [6, 7, 9-11]. However, to date, there has been no evaluation of worldwide research productivity 
associated with SS. The aim of this study was to evaluate the quantity and quality of academic publications associated with SS using the Web of Science (WoS) database. A secondary aim was to present the features of global production in the field of SS. This study will be able of benefit to researchers in obtaining a holistic idea of SS and can be of guidance for future studies.

\section{Materials and methods}

The methodology of this study was based on similar bibliometric analysis in the literature [12, 13]. Data were collected from the WoS database on May 12, 2020. WoS is the world's leading database of scientific publications. Citations and other academic impact information can be obtained from the WoS database. This feature makes WoS a frequently used resource for bibliometric studies and scientific productivity researches. WoS provides ease of use, presents simple and advanced search tools. WoS covers highly cited and influential journals and has long established the standard for determining which citations are counted. WoS is more selective and strict; it is more demanding to index in its body. With strict inclusion criteria, WoS presents adequate studies for literature analysis. Therefore, WoS was used instead of other databases (Scopus, Google Scholar, Pubmed etc.) in this study. Additionally, WoS database was widely used in studies with similar methodology [14-17]. The WoS Core Collection was used to obtain the data. Topic search was selected and articles were listed using the search terms 'Sjögren's syndrome' and 'Sjögren syndrome' for the period of January 2010-December 2019. Such a path was followed, as both 'Sjögren's syndrome' and 'Sjögren syndrome' were used in the literature. Original articles and reviews were selected for further analyses as in similiar bibliometric studies in the literature $[13,15,18]$. Meeting abstracts, letters, editorial materials, case reports, books, book chapters, corrections, proceedings papers and other publication types were excluded from this study.

The total number of articles, year of article, country of article and citation data of article were recorded [12]. Journals indexed in the Science Citation Index Expanded (SCIE) and Emerging Sources Citation Index (ESCI) were noted separately. The total number of articles for each year (from 2010 to 2019) was calculated. As in studies with similar methodology, when the authors were from different countries, the country of corresponding author was taken into consideration and accepted as the country of article [12]. The number of articles was analyzed considering the population and gross domestic product (GDP) of the country. The population and GDP data were obtained from 'https ://www.cia.gov/library/publicaitons/the-world-factbook'. The total number of articles and citations for each country were recorded. The total number of citations was divided by the total number of articles to calculate the average number of citations [19, 20]. Thus, average number of citations was calculated for each country. The academic productivity of the countries was quantitatively evaluated according to the number of articles. Citation data were also assessed. Countries were categorised in four groups as high-income, upper middle-income, low middle-income and low-income, according to World Bank data [21].

Countries that were the source of at least $1 \%$ of the total number of articles in SS were accepted as the main active countries $[15,19,22]$. The contribution rates of countries were calculated by dividing the number of articles of each country by the total number of articles. The top five countries in terms of the total number of articles were deemed to be the 'five most productive countries'. The top five journals in terms of the total number of articles published were accepted as 'five active journals' [12]. The five most productive countries in the five most active journals were determined and listed.

\section{Ethics statement}

No human participants/animals were enrolled. The data were provided from the Web of Science database so that ethics committee approval was not required for this study.

\section{Statistical analysis}

The Statistical Package for the Social Sciences version 20.0 package program (SPSS Inc., Chicago, IL, USA) was used to analyze the results of the bibliometric analysis. Data were stated as number $(n)$ and percentage (\%). The yearly trends of SS articles from all over the world and the top five countries between 2010 and 2019 were evaluated using regression analysis. A value of $p<0.05$ was accepted as statistically significant.

\section{Results}

Using the search term, "Sjögren's syndrome", 6134 publications were identified in the 10-year period of 2010 to 2019. Using the search term, "Sjögren syndrome", 7686 publications were detected in the same date range. From these, original articles and reviews were selected, and articles not related to SS, articles related to Marinesco-Sjogren syndrome and Sjogren-Larsson syndrome were excluded. Additionally, publications listed in both search terms were evaluated once. Finally, a total of 3856 articles from 65 different countries were included for analysis. A statistically significant trend was detected in the direction of increase (in terms of the number of articles; from 282 to 461) in 
SS-related articles $(p<0.001)$. A total of $3004(77.90 \%)$ articles were sourced from high-income countries, $781(20.25 \%)$ from upper middle-income countries and $71(1.85 \%)$ from lower middle-income countries. No articles originated from a low-income country.

The five most productive countries were the United States $(n=714,18.51 \%)$, China $(n=428,11.09 \%)$, Japan

Table 1 Yearly number of articles from the five most productive countries between 2010 and 2019

\begin{tabular}{llllll}
\hline Year & $\begin{array}{l}\text { United } \\
\text { states }(n)\end{array}$ & China $(n)$ & Japan $(n)$ & Italy $(n)$ & France $(n)$ \\
\hline 2010 & 50 & 11 & 32 & 20 & 33 \\
2011 & 46 & 19 & 20 & 15 & 17 \\
2012 & 66 & 21 & 37 & 26 & 21 \\
2013 & 58 & 40 & 28 & 28 & 23 \\
2014 & 84 & 49 & 28 & 30 & 23 \\
2015 & 96 & 50 & 41 & 42 & 29 \\
2016 & 96 & 42 & 29 & 24 & 28 \\
2017 & 81 & 60 & 27 & 33 & 27 \\
2018 & 89 & 82 & 47 & 38 & 21 \\
2019 & 62 & 64 & 19 & 43 & 26 \\
\hline
\end{tabular}

$n$ number of articles $(n=308,7.98 \%)$, Italy $(n=299,7.75 \%)$ and France $(n=249$, $6.45 \%)$. When these countries were analyzed according to the number of articles in the ten-year period, a significant growth trend was detected in the United States, China and Italy $(p<0.05)$. No significant change was detected in Japan and France $(p>0.05)$. Over the years, the number of articles originating from China approached the number of articles originating from the United States and exceeded this by a minimal difference in 2019 (Table 1). However, the articles from the United States had a higher number of total citations and average citations than those from China (15597 vs. $4712 ; 21.84$ vs. 11.00 ) (Table 2).

The contribution rate values of the five most productive countries over the years were calculated. An increase in contribution rate was found in China (from $3.88 \%$ to $13.88 \%$ ) and Italy (from $7.09 \%$ to $9.32 \%$ ). A decrease in contribution rate was detected in the United States (from $17.73 \%$ to $13.44 \%$ ), Japan (from $11.34 \%$ to $4.12 \%$ ) and France (from $11.70 \%$ to $5.63 \%$ ) (Table 3 ).

A total of 21 countries met the main active country criteria. These 21 countries were the source of the vast majority of articles (89.62\%) (Table 2). Of the main active countries, 17 were in the high-income country group, 3 were in the upper middle-income country group (China, Turkey, Mexico), and 1 was in the lower middle-income country group
Table 2 The main active countries in SS-related articles between 2010 and 2019

\begin{tabular}{llrrll}
\hline Country & $n(\%)$ & \multicolumn{1}{c}{$n^{\mathrm{a}}$} & \multicolumn{1}{c}{$n^{\mathrm{b}}$} & Total citations & Average citations \\
\hline United States & $714(18.51)$ & 2.15 & 3.66 & 15597 & 21.84 \\
China & $428(11.09)$ & 0.30 & 1.68 & 4712 & 11.00 \\
Japan & $308(7.98)$ & 2.45 & 5.66 & 5004 & 16.24 \\
Italy & $299(7.75)$ & 4.79 & 12.94 & 6287 & 21.02 \\
France & $249(6.45)$ & 3.67 & 8.73 & 6376 & 25.60 \\
South Korea & $147(3.81)$ & 2.83 & 7.24 & 1544 & 10.50 \\
United Kingdom & $145(3.76)$ & 2.20 & 4.96 & 2916 & 20.11 \\
Netherlands & $125(3.24)$ & 7.23 & 13.58 & 2452 & 19.61 \\
Spain & $124(3.21)$ & 2.49 & 7.06 & 2269 & 18.29 \\
Germany & $122(3.16)$ & 1.52 & 2.98 & 1744 & 14.29 \\
Greece & $110(2.85)$ & 10.37 & 37.93 & 2906 & 26.41 \\
Turkey & $108(2.80)$ & 1.31 & 4.95 & 866 & 10.17 \\
Taiwan & $101(2.61)$ & 4.27 & 8.55 & 1028 & 10.20 \\
Brazil & $91(2.35)$ & 0.42 & 2.80 & 929 & 20.47 \\
Norway & $85(2.20)$ & 15.56 & 22.36 & 1740 & 21.40 \\
Sweden & $72(1.86)$ & 7.05 & 14.11 & 1541 & 26.18 \\
Australia & $54(1.40)$ & 2.12 & 4.35 & 1414 & 20.92 \\
Canada & $50(1.29)$ & 1.32 & 2.82 & 1046 & 8.24 \\
Mexico & $45(1.16)$ & 0.34 & 1.82 & 371 & 1345 \\
Israel & $42(1.08)$ & 4.84 & 13.54 & 178 & \\
India & $41(1.06)$ & 0.03 & 0.43 & 178 & \\
\hline
\end{tabular}

$n$ number of articles, \% percentage, SS Sjögren's syndrome

$n^{\text {a }}$ number of articles per million population

$n^{\mathrm{b}}$ number of articles per $\$ 100$ billion gross domestic product 
Table 3 Contribution rate of the five most productive countries between 2010 and 2019

\begin{tabular}{llrrrc}
\hline Year & $\begin{array}{l}\text { United states } \\
(\%)\end{array}$ & China (\%) & Japan (\%) & Italy (\%) & France (\%) \\
\hline 2010 & 17.73 & 3.88 & 11.34 & 7.09 & 11.70 \\
2011 & 17.76 & 7.33 & 7.72 & 5.79 & 6.56 \\
2012 & 19.18 & 6.10 & 10.75 & 7.55 & 6.10 \\
2013 & 19.46 & 13.42 & 9.39 & 9.39 & 7.71 \\
2014 & 21.87 & 10.15 & 7.29 & 7.81 & 5.98 \\
2015 & 21.69 & 11.79 & 9.66 & 9.90 & 6.83 \\
2016 & 20.77 & 9.09 & 6.27 & 5.19 & 6.27 \\
2017 & 18.24 & 13.51 & 6.08 & 7.43 & 6.08 \\
2018 & 17.87 & 16.46 & 9.43 & 7.63 & 4.21 \\
2019 & 13.44 & 13.88 & 4.12 & 9.32 & 5.63 \\
\hline
\end{tabular}

$\%$ percentage

(India). When the number of articles was calculated per million population, Norway (15.56) was the most productive, followed by Greece (10.37) and the Netherlands (7.23). In calculations performed according to GDP, Greece was the most productive (37.93), followed by Norway (22.36) and the Netherlands (13.58). In the assessment of total citations, the United States had the highest number of citations (15597), followed by France (6376) and Italy (6287). According to the average number of citations, Israel (32.02), Greece (26.41) and Australia (26.18) were in the top three (Table 2).

A total of $3641(94.42 \%)$ articles were published in journals indexed in SCIE. The total citations and average citations of these articles were 65726 and 18.05 , respectively. A total of $2873(78.90 \%)$ articles were sourced from high-income countries, 720 (19.78\%) from upper middleincome countries and 48 (1.32\%) from lower middle-income countries.

A total of 215 (5.58\%) articles were published in journals indexed in ESCI. The total citations and average citations of these articles were 893 and 4.15, respectively. A total of $131(60.93 \%)$ articles were sourced from highincome countries, 61 (28.37\%) from upper middle-income countries and $23(10.70 \%)$ from lower middle-income countries.

The five most active journals were evaluated in the five most productive countries (United States, China, Japan, Italy, and France). Arthritis \& Rheumatology (AR) ranked first in the United States, Clinical Rheumatology (CR) ranked first in China, Modern Rheumatology (MR) ranked first in Japan, Clinical and Experimental Rheumatology (CER) ranked first in Italy, and Reveu- De Medicine Interne (RMI) ranked first in France (Table 4).

The top five journals were listed according to the number of articles published on SS. CER was in the first place $(n=140,3.63 \%)$, followed by Rheumatology $(n=105$, $2.72 \%)$, CR $(n=100,2.59 \%)$, Plos One $(n=94,2.43 \%)$ and Journal of Autoimmunity (JA) $(n=90,2.33 \%)$, respectively. The top five countries in these five most active journals are presented in Table 5. Italy, Italy, China, USA and Greece ranked first in CER, Rheumatology, CR, Plos One, JA, respectively, and the United States was on the list of all five journals.

The top five journals indexed in ESCI were listed according to the number of articles. RMD Open was in the first place $(n=16,7.44 \%)$, followed by Reumatologia Clinica $(n=11,5.11 \%)$, Current Rheumatology Reviews $(n=8,3.72 \%)$, Clinical Ophthalmology $(n=8,3.72 \%)$ and Revista Cubana de Reumatologia $(n=6,2.79 \%)$.

After the exclusion criteria, it was determined that 81 $(2.10 \%)$ articles were published in Rheumatology Internatonal (RI). Average number of citations per article was calculated as 11.14 for RI. This value was calculated as 11.90 for CER, 23.22 for Rheumatology, 6.99 for CR, 17.09 for Plos One and 39.25 for JA. Articles originating from 29 different countries were determined. The top five countries in RI were Turkey $(n=9,11.11 \%)$, South Korea $(n=9,11.11 \%)$, Spain $(n=6,7.40 \%)$, China $(n=5,6.17 \%)$ and Japan $(n=4,4.93 \%)$.
Table 4 The five most active journals in the five most productive countries

\begin{tabular}{llllll}
\hline Rank & United states $(n)$ & China $(n)$ & Japan $(n)$ & Italy $(n)$ & France $(n)$ \\
\hline 1 & AR (23) & CR (38) & MR (45) & CER (40) & RMI (18) \\
2 & Plos one (21) & IJRD (15) & Plos one (11) & AI (16) & JBS (16) \\
3 & CI (20) & Medicine (14) & CEI (10) & Rheumatology (16) & JA (16) \\
4 & IOVS (18) & Plos one (10) & IJMS (8) & ART (11) & ARD (14) \\
5 & ART (16) & CER (8) & ART (7) & JA (8) & Medicine (10) \\
\hline
\end{tabular}

$n$ number of articles, $A \& R$ Arthritis \& Rheumatology, CI Clinical Immunology, IOVS Investigative Ophthalmology \& Visual Science, ART Arthritis Research \& Therapy, CR Clinical Rheumatology, IJRD International Journal of Rheumatic Diseases, CER Clinical and Experimental Rheumatology, MR Modern Rheumatology, CEI Clinical \& Experimental Immunology, IJMS International Journal of Molecular Sciences, ART Arthritis Research \& Therapy, AI Autoimmunity Reviews, JA Journal of Autoimmunity, RMI Reveu-De Medicine Interne, JBS Joint Bone Spine, ANR Annals of the Rheumatic Diseases 
Table 5 Top five countries in the five most active journals

\begin{tabular}{llllll}
\hline Rank & CER $(n)$ & Rheumatology $(n)$ & CR $(n)$ & Plos one $(n)$ & JA $(n)$ \\
\hline 1 & Italy (40) & Italy (16) & China (38) & United States (21) & Greece (26) \\
2 & United States (13) & United Kingdom (15) & United States (7) & Japan (11) & France (16) \\
3 & Spain (12) & Netherlands (11) & Taiwan (6) & China (10) & Italy (8) \\
4 & Netherlands (10) & United States (11) & Brazil (5) & Taiwan (8) & United States (7) \\
5 & South Korea (10) & France (9) & Turkey (5) & Norway (6) & Netherlands (6) \\
\hline
\end{tabular}

$n$ number of articles, CER Clinical and Experimental Rheumatology, CR Clinical Rheumatology, JA Journal of Autoimmunity

\section{Discussion}

The productivity trends on different topics should be determined to ensure medical/academic development and proper resource management. Bibliometric analysis can be utilized to assess publication productivity and reveal contributions from different parts of the world. This is the first study to use bibliometric analysis to evaluate SS articles globally. A significant increase in the number of SS articles was detected between 2010 and 2019. More than three in four of the articles originated from high-income countries. The United States ranked first in the total number of articles and citations. Over the years, China approached the United States in terms of the number of articles and exceeded that number in 2019. Considering the citation data, it is clear that China should also improve publication quality. European countries were at the forefront when the analysis was performed according to GDP and population.

According to the total number of articles, the United States, China, Japan, Italy and France were in the five most productive countries. Thus, it can be considered that the United States has a prominent role in the SS field. This result is not surprising as it has been revealed that the United States is the most productive country in many different topics [14, 19, 23-25]. However, China has increased publication productivity in recent years and passed the United States in 2019. China's population can be a factor in this situation, as a larger population will have a greater number of researchers and patients. In parallel with the development in the Chinese economy, increased financial support for medical research may be another reason [26]. According to these data, it can be predicted that China will have an increasing impact on global academic productivity in the near future.

The United States was seen to be the country with the highest total number of citations, which can be attributed to it also being the country with the highest total number of articles. Considering the difference between the United States and China, it can be accepted that not only the number of articles but also high quality increased the number of citations. Although China had the second highest total number of articles, it was in fifth place in the total number of citations. These results demonstate that China should make more effort to increase research quality.

In various bibliometric studies, the productivity rates of high and non-high-income countries have been evaluated and a ratio of $9 / 1$ has been defined in favor of high-income countries. In the current study, although more than three quarters of the articles were from high-income countries, an increase was detected in the number of articles sourced from middle-income countries [19, 27]. China, Brazil, Turkey, Mexico and India in the main active country group led to this result, with increased publication production from China in particular. Problems in the publication contribution of low-income countries can be attributed to medical policies, research funds, and the number of researchers.

When the results were adjusted according to population and GDP, European countries including Norway, Greece and the Netherlands came to the fore. These results suggest that relative productivity is very high in these countries, and it can also be concluded that these European countries have used financial and human resources efficiently. Israel, Greece and Australia ranked in the top three according to the average number of citations. It was noteworthy that China was not at the forefront in terms of these data, thereby demonstrating that the three aforementioned countries produced relatively few but high-quality articles.

Most of the articles were published in journals indexed in SCIE. There was a notable difference in the average number of citations in favor of articles published in journals indexed in SCIE (18.05 vs. 4.15). This result suggests that journals indexed in ESCI should be more selective and have a long way to go in terms of quality. Considering the journals indexed in SCIE, a decrease was detected in the rate of high-income country originated articles in journals indexed in ESCI. In addition, there was an increase in the rate of articles from upper middle-income and lower middleincome countries in journals indexed in ESCI. The fact that researchers from upper middle-income and lower middleincome countries submit articles that cannot be published in SCIE journals to ESCI journals may explain this result.

The five most active journals were assessed in the five productive countries. AR ranked first in the United States, CR ranked first in China, MR ranked first in Japan, CER 
ranked first in Italy, and RMI ranked first in France. These results suggest that journals tend to publish articles from the countries in which they are located. Examples from AR-United States, MR - Japan, CER-Italy and RMIFrance support this view. This may be due to the tendency of researchers to submit articles to journals in their region, and previous habits.

The top five journals were CER, Rheumatology, CR, Plos One and JA, respectively. It showed that these journals had a major role in the development and sharing of literature related to SS. The top five countries in these five most active journals were evaluated. Italy, Italy, China, USA and Greece ranked first in CER, Rheumatology, CR, Plos One, JA, respectively. The United States was on the list of all five journals. These results revealed the influence of the United States and Italy in the top five journals.

This study had some limitations arising from bibliometric study methodology. Non-English language publications were not included in the study. Even if written in English, publications not listed in the WoS database could not be evaluated. Therefore, the results may change in research conducted on a different database, such as Scopus or Google Scholar. The reasons for choosing WoS as a data source were that it is the most reliable database for citations and it has been used commonly in bibliometric studies in the literature. Different results may be observed from bibliometric analysis performed at a different time interval. Self-citation could not be evaluated. Despite these limitations, taking into account the objective and comprehensive methodology, this study can still be considered to provide a correct view of the global publication productivity associated with SS.

\section{Conclusion}

In the yearly evaluation, the number of SS articles tended to increase rapidly between 2010 and 2019. More than three quarters of the articles were provided from high-income countries. Although the United States was the most productive country, China's rise should not be overlooked. The number of articles from China exceeded the United States in 2019, but it is clear that China should make more effort to improve publication quality. Taking population and GDP into consideration, relatively small European countries came to the fore.

\footnotetext{
Author contributions AA and BFK contributed substantial contributions to the conception and design of the work; the acquisition, analysis, and interpretation of data for the work. AA and BFK made drafting for the work and revising it critically for important intellectual content and made final approval of the version to be published. AA and BFK contributed to agreement to be accountable for all aspects of the work in ensuring that the questions related to the accuracy or integrity of
}

any part of the work are appropriately investigated and resolved. AA and BFK are familiar and take full responsibility for all aspects of the study and the final manuscript.

\section{Compliance with ethical standards}

Conflict of interest The authors declare no conflicts of interest.

Human and animal rights No human participants/animals were enrolled. The data were provided from the Web of Science database so that ethics committee approval was not required for this study.

\section{References}

1. Odani T, Chiorini JA (2019) Targeting primary Sjögren's syndrome. Mod Rheumatol 29:70-86. https://doi.org/10.1080/14397 595.2018 .1546268

2. Vivino FB (2017) Sjogren's syndrome: clinical aspects. Clin Immunol 182:48-54. https://doi.org/10.1016/j.clim.2017.04.005

3. Chen X, Wu H, Wei W (2018) Advances in the diagnosis and treatment of Sjogren's syndrome. Clin Rheumatol 37:1743-1749. https://doi.org/10.1007/s10067-018-4153-8

4. Cornec D, Chiche L (2015) Is primary Sjögren's syndrome an orphan disease? A critical appraisal of prevalence studies in Europe. Ann Rheum Dis 74:e25. https://doi.org/10.1136/annrh eumdis-2014-206860

5. Patel R, Shahane A (2014) The epidemiology of Sjögren's syndrome. Clin Epidemiol 6:247-255. https://doi.org/10.2147/CLEP. S47399

6. Jamshidi AR, Gharibdoost F, Nadji A, Nikou M, Habibi G, Mardani A, Ghaemi M (2013) Presentation of psoriatic arthritis in the literature: a twenty-year bibliometric evaluation. Rheumatol Int 33:361-367. https://doi.org/10.1007/s00296-012-2428-y

7. Elango B, Rajendran P, Bornmann L (2013) Global nanotribology research output (1996-2010): a scientometric analysis. PLoS ONE 8:e81094. https://doi.org/10.1371/journal.pone.0081094

8. Mansoori P (2018) 50 years of Iranian clinical, biomedical, and public health research: a bibliometric analysis of the web of science core collection (1965-2014). J Glob Health 8:020701. https ://doi.org/10.7189/jogh.08.020701

9. Şenel E, Demir E (2018) Bibliometric analysis of apitherapy in complementary medicine literature between 1980 and 2016. Complement Ther Clin Pract 31:47-52. https://doi.org/10.1016/j. ctcp.2018.02.003

10. Devos P, Menard J (2019) Bibliometric analysis of research relating to hypertension reported over the period 1997-2016. J Hypertens 37:2116-2122. https://doi.org/10.1097/HJH.0000000000 002143

11. Müller AM, Ansari P, Ebrahim NA, Khoo S (2016) Physical activity and aging research: a bibliometric analysis. J Aging Phys Act 24:476-483. https://doi.org/10.1123/japa.2015-0188

12. Zhao X, Chen J, Pan Y, Feng H, Meng B, Meng Y (2019) A bibliometric analysis of the global research in ankylosing spondyloarthritis (2008-2017). Rheumatol Int 39:1091-1097. https:// doi.org/10.1007/s00296-019-04308-6

13. Lei J, Zhao X, Xu B, Duan Z, Shen Z, Yang H, Jiao G, Ouyang Q, Tian J (2018) Global scientific productivity in the field of PET: a 10-year survey of research activities. Nucl Med Commun 39:277-282. https://doi.org/10.1097/MNM.0000000000000805

14. Şenel E, Demir E, Alkan RM (2017) Bibliometric analysis on global Behçet disease publications during 1980-2014: is there 
a Silk Road in the literature? J Eur Acad Dermatol Venereol 31:518-522. https://doi.org/10.1111/jdv.13897

15. Zhang J, Chen X, Gao X, Yang H, Zhen Z, Li Q, Lin Y, Zhao X (2017) Worldwide research productivity in the field of psychiatry. Int J Ment Health Syst 11:20. https://doi.org/10.1186/s1303 3-017-0127-5

16. Cheng T, Zhang G (2013) Worldwide research productivity in the field of rheumatology from 1996 to 2010: a bibliometric analysis. Rheumatol (Oxford) 52:1630-1634. https://doi.org/10.1093/ rheumatology/ket008

17. O'Neill CJ, Cassar-Gheiti AJ, Harty JA (2019) Arthroplasty and global research output: a bibliometric analysis. J Orthop 17:187192. https://doi.org/10.1016/j.jor.2019.06.017

18. Tao Z, Zhou S, Yao R, Wen K, Da W, Meng Y, Yang K, Liu H, Tao L (2020) COVID-19 will stimulate a new coronavirus research breakthrough: a 20-year bibliometric analysis. Ann Transl Med 8:528. https://doi.org/10.21037/atm.2020.04.26

19. Zhao X, Ye R, Zhao L, Lin Y, Huang W, He X, Lian F, Tong X (2015) Worldwide research productivity in the field of endocrinology and metabolism-a bibliometric analysis. Endokrynol Pol 66:434-442. https://doi.org/10.5603/EP.2015.0054

20. Zhang C, Feng X, Wang C, Liu D, He C, Xu W (2019) Bibliometric analysis of scientific publications in rheumatology journals from China and other top-ranking countries between 2007 and 2017. PeerJ 7:e6825. https://doi.org/10.7717/peerj.6825

21. https://datahelpdesk.worldbank.org/knowledgebase/articles/90651 9-world-bank-country-and-lending-groups. Accessed June 2020

22. Wang B, Zhao P (2018) Worldwide research productivity in the field of back pain: a bibliometric analysis. Med (Baltim) 97:e11566. https://doi.org/10.1097/MD.0000000000011566
23. Liang Z, Luo X, Gong F, Bao H, Qian H, Jia Z, Li G (2015) Worldwide research productivity in the field of arthroscopy: a bibliometric analysis. Arthroscopy 31:1452-1457. https://doi. org/10.1016/j.arthro.2015.03.009

24. Qi B, Jin S, Qian H, Zou Y (2020) Bibliometric analysis of chronic traumatic encephalopathy research from 1999 to 2019. Int J Environ Res Public Health 17:5411. https://doi.org/10.3390/ ijerph17155411

25. Zhang T, Yin X, Yang X, Man J, He Q, Wu Q, Lu M (2020) Research trends on the relationship between microbiota and gastric cancer: a Bibliometric Analysis from 2000 to 2019. J Cancer 11:4823-4831. https://doi.org/10.7150/jca.44126

26. Wang C, Liu Q (2013) A turning point for clinical research in China? Lancet 382:835-836. https://doi.org/10.1016/S0140 $-6736(13) 61804-8$

27. Saxena S, Paraje G, Sharan P, Karam G, Sadana R (2006) The 10/90 divide in mental health research: trends over a 10-year period. Br J Psychiatry 188:81-82. https://doi.org/10.1192/bjp. bp. 105.011221

Publisher's Note Springer Nature remains neutral with regard to jurisdictional claims in published maps and institutional affiliations. 